Nonlinear Analysis: Real World Applications, Vol. 10, № 1, 416-427 (2009)

\title{
Solution of an anti-symmetric quadratic nonlinear oscillator by a modified He's homotopy perturbation method
}

\author{
A. Beléndez, C. Pascual, T. Beléndez and A. Hernández \\ Departamento de Física, Ingeniería de Sistemas y Teoría de la Señal. \\ Universidad de Alicante. Apartado 99. E-03080 Alicante. SPAIN \\ E-mail: a.belendez@ua.es
}

Corresponding author: A. Beléndez

Phone: +34-96-5903651

Fax: $+34-96-5909750$ 


\begin{abstract}
In this paper He's homotopy perturbation method has been adapted to calculate higherorder approximate periodic solutions of a nonlinear oscillator with discontinuity for which the elastic force term is anti-symmetric and quadratic term. We find He's homotopy perturbation method works very well for the whole range of initial amplitudes, and the excellent agreement of the approximate frequencies and periodic solutions with the exact ones has been demonstrated and discussed. Only one iteration leads to high accuracy of the solutions with a maximal relative error for the approximate period of less than $0.73 \%$ for all values of oscillation amplitude, while this relative error is as low as $0.040 \%$ when the second iteration is considered. Comparison of the result obtained using this method with those obtained by harmonic balance method reveals that the former is very effective and convenient.
\end{abstract}

Keywords: Nonlinear oscillator; Approximate solutions; Homotopy perturbation method. 


\section{Introduction}

The study of nonlinear problems is of crucial importance not only in all areas of physics but also in engineering and other disciplines, since most phenomena in our world are essentially nonlinear and are described by nonlinear equations. It is very difficult to solve nonlinear problems and, in general, it is often more difficult to get an analytic approximation than a numerical one for a given nonlinear problem. There are several methods used to find approximate solutions to nonlinear problems, such as perturbation techniques [1-6], harmonic balance based methods [6-9] or other techniques [10-18]. An excellent review on some asymptotic methods for strongly nonlinear equations can be found in detail in references [19] and [20].

In the present paper we obtain higher-order analytical approximations to the periodic solutions to a nonlinear oscillator with discontinuity for which the elastic restoring force is an anti-symmetric quadratic nonlinear oscillator. This is a nonlinear oscillator with a discontinuity. To do this, we apply He's homotopy perturbation method [21-39]. Recently, a considerable amount of attention has been directed towards analytical solutions for non-linear equations without possible small parameters [40]. To eliminate this disadvantage $\mathrm{He}$ adopted the homotopy technique which is widely applied in differential topology for obtaining an approximate analytic solution of nonlinear differential equations. The homotopy perturbation method, a coupling of the traditional perturbation method and homotopy in topology, deforms continuously a difficult problem to a simple problem which is easily solved.

This nonlinear oscillator was analyzed by He applying the first-order homotopy perturbation method [21], however no higher-order analytical approximate solutions have been obtained for this oscillator using He's method. As we can see, the results presented in this paper reveal that the method is very effective and convenient for conservative nonlinear oscillators for which the restoring force has a non-polynomial form. 


\section{Solution procedure}

This paper considers the following nonlinear oscillator with discontinuity

$$
\frac{\mathrm{d}^{2} x}{\mathrm{~d} t^{2}}+x|x|=0
$$

with initial conditions

$$
x(0)=A \quad \text { and } \quad \frac{\mathrm{d} x}{\mathrm{~d} t}(0)=0
$$

This is a conservative system and all the solutions to Eq. (1) are periodic [6, 41]. There exists no small parameter in the equation. Therefore, the traditional perturbation method cannot be applied directly. Due to the fact that the homotopy perturbation method requires neither a small parameter nor a linear term in a differential equation, we can approximately solve Eq. (1) using the homotopy perturbation method. In this method, an artificial perturbation equation is constructed by embedding an artificial parameter $p \in[0,1]$, which is used as expanding parameter. This technique yields a very rapid convergence of the solution series; in most cases, only one iteration leads to high accuracy of the solution.

In order to use homotopy perturbation method, we re-write Eq. (1) in the following form

$$
\frac{\mathrm{d}^{2} x}{\mathrm{~d} t^{2}}+x=x-x|x|
$$

For Eq. (3) we can establish the following homotopy

$$
\frac{\mathrm{d}^{2} x}{\mathrm{~d} t^{2}}+x=p(x-x|x|)
$$

where $p$ is the homotopy parameter. When $p=0$, Eq. (4) becomes a linear differential equation for which an exact solution can be calculated; for $p=1$, Eq. (4) then becomes the 
original problem. Now the homotopy parameter $p$ is used to expand the solution $x(t)$ and the square of the unknown angular frequency $\omega$ as follows

$$
\begin{gathered}
x(t)=x_{0}(t)+p x_{1}(t)+p^{2} x_{2}(t)+\ldots \\
1=\omega^{2}-p \alpha_{1}-p^{2} \alpha_{2}-\ldots
\end{gathered}
$$

where $\alpha_{i}(i=1,2, \ldots)$ are to be determined.

Substituting Eqs. (5) and (6) into Eq. (4)

$$
\begin{aligned}
& \left(x_{0}^{\prime \prime}+p x_{1}^{\prime \prime}+p^{2} x_{2}^{\prime \prime}+\ldots\right)+\left(\omega^{2}-p \alpha_{1}-p^{2} \alpha_{2}-\ldots\right)\left(x_{0}+p x_{1}+p^{2} x_{2}+\ldots\right)= \\
& \quad=p\left[\left(x_{0}+p x_{1}+p^{2} x_{2}+\ldots\right)-\left(x_{0}+p x_{1}+p^{2} x_{2}+\ldots\right)\left|x_{0}+p x_{1}+p^{2} x_{2}+\ldots\right|\right]
\end{aligned}
$$

and collecting the terms of the same power of $p$, we obtain a series of linear equations, of which we write only the first four

$$
\begin{array}{ll}
x_{0}^{\prime \prime}+\omega^{2} x_{0}=0, & x_{0}(0)=A, x_{0}^{\prime}(0)=0 \\
x_{1}^{\prime \prime}+\omega^{2} x_{1}=\left(1+\alpha_{1}\right) x_{0}-x_{0}\left|x_{0}\right|, & x_{1}(0)=x_{1}^{\prime}(0)=0 \\
x_{2}^{\prime \prime}+\omega^{2} x_{2}=\alpha_{2} x_{0}+\left(1+\alpha_{1}\right) x_{1}-2\left|x_{0}\right| x_{1}, & x_{2}(0)=x_{2}^{\prime}(0)=0 \\
x_{3}^{\prime \prime}+\omega^{2} x_{3}=\alpha_{3} x_{0}+\left(1+\alpha_{2}\right) x_{1}+\alpha_{1} x_{2}-2\left|x_{0}\right| x_{2}-\operatorname{sgn}\left(x_{0}\right) x_{1}^{2}, & x_{3}(0)=x_{3}^{\prime}(0)=0
\end{array}
$$

In Eqs. (8)-(10) we have taken into account the following expression 


$$
\begin{array}{r}
f(x)=f\left(x_{0}+p x_{1}+p^{2} x_{2}+p^{3} x_{3}+\ldots\right)=f\left(x_{0}\right)+p\left(\frac{\mathrm{d} f(x)}{\mathrm{d} x}\right)_{x=x_{0}} x_{1}+ \\
+p^{2}\left[\left(\frac{\mathrm{d} f(x)}{\mathrm{d} x}\right)_{x=x_{0}} x_{2}+\frac{1}{2}\left(\frac{\mathrm{d}^{2} f(x)}{\mathrm{d} x^{2}}\right)_{x=x_{0}} x_{1}^{2}\right]+O\left(p^{3}\right)
\end{array}
$$

where $f(x)=x|x|$ and

$$
\begin{gathered}
\frac{\mathrm{d}(x|x|)}{\mathrm{d} x}=|x|+x \frac{\mathrm{d}|x|}{\mathrm{d} x}=|x|+x \operatorname{sgn}(x)=2|x| \\
\frac{\mathrm{d}^{2}(x|x|)}{\mathrm{d} x^{2}}=\frac{\mathrm{d}(2|x|)}{\mathrm{d} x}=2 \operatorname{sgn}(x) \\
\frac{\mathrm{d}^{3}(x|x|)}{\mathrm{d} x^{3}}=\frac{\mathrm{d}^{4}(x|x|)}{\mathrm{d} x^{4}}=\ldots=0
\end{gathered}
$$

The solution of Eq. (8) is

$$
x_{0}(t)=A \cos \omega t
$$

Substitution of this result into the right side of Eq. (9) gives

$$
x_{1}^{\prime \prime}+\omega^{2} x_{1}=\left(1+\alpha_{1}\right) A \cos \omega t-A^{2} \cos \omega t|\cos \omega t|
$$

It is possible to do the following Fourier series expansion

$$
\cos \omega t|\cos \omega t|=\sum_{n=0}^{\infty} a_{2 n+1} \cos [(2 n+1) \omega t]=a_{1} \cos \omega t+a_{3} \cos 3 \omega t+\ldots
$$

where

$$
a_{2 n+1}=\frac{4}{\pi} \int_{0}^{\pi / 2} \cos \tau|\cos \tau| \cos [(2 n+1) \tau] \mathrm{d} \tau=\frac{(-1)^{n} 8}{\left(3+2 n-12 n^{2}-8 n^{3}\right) \pi}
$$


The first term of the expansion in Eq. (19) is given by

$$
a_{1}=\frac{8}{3 \pi}
$$

Therefore, the first several terms are

$\cos \omega t|\cos \omega t|$

$$
=a_{1}\left[\cos \omega t+\frac{1}{5} \cos 3 \omega t-\frac{1}{35} \cos 5 \omega t+\frac{1}{105} \cos 7 \omega t-\frac{1}{231} \cos 9 \omega t+\frac{1}{429} \cos 11 \omega t-\ldots\right]
$$

Substituting Eq. (18) into Eq. (17), we have

$$
x_{1}^{\prime \prime}+\omega^{2} x_{1}=\left[\left(1+\alpha_{1}\right) A-a_{1} A^{2}\right] \cos \omega t-A^{2} \sum_{n=1}^{\infty} a_{2 n+1} \cos [(2 n+1) \omega t]
$$

No secular terms in $x_{1}(t)$ requires eliminating contributions proportional to $\cos \omega t$ on the right-hand side of Eq. (22) and we obtain

$$
\alpha_{1}=-1+a_{1} A=-1+\frac{8 A}{3 \pi}
$$

From Eqs. (6) and (23), writing $p=1$, we can easily find that the first-order approximate frequency is

$$
\omega_{1}(A)=\sqrt{\frac{a_{1}}{A}}=\sqrt{\frac{8 A}{2 \pi}}=0.921318 \sqrt{A}
$$

which agrees exactly with Wang and He's solution obtained by first-order parameter expanding method [42], with Mickens's solution obtained by first-order harmonic balance 
method [6] and with He's solution obtained by first-order homotopy perturbation method [21]. However, this paper only considers the first-order approximation.

Now in order to obtain the correction term $x_{1}$ for the periodic solution $x_{0}$ we consider the following procedure. Taking into account Eqs. (22) and (23), we re-write Eq. (22) in the form

$$
x_{1}^{\prime \prime}+\omega^{2} x_{1}=-A^{2} \sum_{n=1}^{\infty} a_{2 n+1} \cos [(2 n+1) \omega t]
$$

with initial conditions $x_{1}(0)=0$ and $x_{1}^{\prime}(0)=0$. The periodic solution to Eq. (25) can be written

$$
x_{1}(t)=\sum_{n=0}^{\infty} b_{2 n+1} \cos [(2 n+1) \omega t]
$$

Substituting Eq. (26) into Eq. (25) we can write the following expression for the coefficients $b_{2 n+1}$

$$
b_{2 n+1}=\frac{a_{2 n+1} A^{2}}{4 n(n+1) \omega^{2}}=\frac{(-1)^{n} 2 A^{2}}{\pi n(n+1)\left(3+2 n-12 n^{2}-8 n^{3}\right) \omega^{2}},
$$

for $n \geq 1$. Taking into account that $x_{1}(0)=0$, Eq. (26) gives

$$
b_{1}=-\sum_{n=1}^{\infty} b_{2 n+1}
$$

To determine the second-order approximate solution it is necessary to substitute Eq. (26) into Eq. (10). Then secular terms are eliminated and parameter $\alpha_{2}$ can be calculated. However, it is difficult to solve the new differential equation because, as $x_{1}(t)$ has an infinite number of harmonics (see Eq. (26)). At this moment we consider a modification in He's homotopy perturbation method [43] to simplify the solution procedure. $x_{1}(t)$ has an infinite number of harmonics, however we can truncate the series expansion at Eq. (26) and write an approximate equation $x_{1}^{(N)}(t)$ in the form 


$$
x_{1}^{(N)}=b_{1}^{(N)} \cos \omega t+\sum_{n=1}^{N} b_{2 n+1} \cos [(2 n+1) \omega t]
$$

where

$$
b_{1}^{(N)}=-\sum_{n=1}^{N} b_{2 n+1}
$$

Equation (29) has only a finite number of harmonics. It is possible to do this approximation because the absolute value of the coefficient $b_{2 n+1}$ decreases when $n$ increases as we can easily verify from Eqs. (21) and (27). Comparing Eqs. (26) and (29), and Eqs. (28) and (30), it follows that

$$
\lim _{N \rightarrow \infty} x_{1}^{(N)}(t)=x_{1}(t), \quad \lim _{N \rightarrow \infty} b_{1}^{(N)}=b_{1}
$$

In the simplest case we consider $N=1(n=0,1)$ in Eqs. (29) and (30), and we obtain

$$
x_{1}^{(1)}(t)=b_{3}(\cos 3 \omega t-\cos \omega t)
$$

From Eq. (27) the following expression for the coefficient $b_{3}$ is obtained

$$
b_{3}=\frac{a_{3} A^{2}}{8 \omega^{2}}=\frac{A^{2}}{15 \pi \omega^{2}}
$$

Therefore, the first approximation to the periodic solution is given

$$
\begin{aligned}
x_{a 1}(t) & =x_{0}\left(t, \omega_{1}\right)+x_{1}^{(1)}\left(t, \omega_{1}\right)=\left(A-b_{3}\right) \cos \left(\omega_{1} t\right)+b_{3} \cos \left(3 \omega_{1} t\right) \\
& =\frac{39}{40} A \cos \left(\omega_{1} t\right)+\frac{1}{40} A \cos \left(3 \omega_{1} t\right)=0.975 A \cos \left(\omega_{1} t\right)+0.025 A \cos \left(3 \omega_{1} t\right)
\end{aligned}
$$

where $\omega_{1}(A)$ is given by Eq. (24). 
Substituting Eqs. (16), (23) and (32) into Eq. (10) gives the following equation for $x_{2}(t)$

$$
x_{2}^{\prime \prime}+\omega^{2} x_{2}=\alpha_{2} A \cos \omega t+\frac{a_{1} a_{3} A^{3}}{8 \omega^{2}}(\cos 3 \omega t-\cos \omega t)-\frac{2 a_{3} A^{3}}{8 \omega^{2}}|\cos \omega t|(\cos 3 \omega t-\cos \omega t)
$$

It is possible to do the following Fourier series expansion

$$
2|\cos \omega t|(\cos 3 \omega t-\cos \omega t)=\sum_{n=0}^{\infty} c_{2 n+1} \cos [(2 n+1) \omega t]=c_{1} \cos \omega t+c_{3} \cos 3 \omega t+\ldots
$$

where

$$
c_{2 n+1}=\frac{4}{\pi} \int_{0}^{\pi / 2} 2|\cos \tau|(\cos 3 \tau-\cos \tau) \cos [(2 n+1) \tau] \mathrm{d} \tau=\frac{(-1)^{n} 64}{\left(-15-26 n+12 n^{2}+8 n^{3}\right) \pi}
$$

The first term in Eq. (37) is given by

$$
c_{1}=-\frac{64}{15 \pi}
$$

Therefore, the first several terms are

$$
\begin{aligned}
& 2|\cos \omega t|(\cos 3 \omega t-\cos \omega t) \\
& \quad=c_{1}\left[\cos \omega t-\frac{5}{7} \cos 3 \omega t-\frac{1}{3} \cos 5 \omega t+\frac{5}{77} \cos 7 \omega t-\frac{1}{39} \cos 9 \omega t+\frac{1}{77} \cos 11 \omega t-\ldots\right]
\end{aligned}
$$

The secular term in the solution for $x_{2}(t)$ can be eliminated if

$$
\alpha_{2} A-\frac{a_{1} a_{3} A^{3}}{8 \omega^{2}}-\frac{a_{3} c_{1} A^{3}}{8 \omega^{2}}=0
$$


Equation (40) can be solved for $\alpha_{2}$, that is

$$
\alpha_{2}=\frac{a_{3}\left(a_{1}+c_{1}\right) A^{2}}{8 \omega^{2}}
$$

From Eqs. (6), (19) and (38), and taking $p=1$, one can easily obtain the following expression for the second-order approximate frequency is

$$
\omega_{2}(A)=\sqrt{\frac{a_{1}}{2 A}+\sqrt{\frac{2 a_{1}^{2}+a_{1} a_{3}+a_{3} c_{1}}{8 A^{2}}}}=\sqrt{\frac{20+2 \sqrt{94}}{15 \pi}} A=0.914274 \sqrt{A}
$$

With the requirement of Eq. (41), we can re-write Eq. (35) in the form

$$
x_{2}^{\prime \prime}+\omega^{2} x_{2}=\frac{a_{1} a_{3} A^{3}}{8 \omega^{2}} \cos 3 \omega t-\frac{a_{3} A^{3}}{8 \omega^{2}} \sum_{n=1}^{\infty} c_{2 n+1} \cos [(2 n+1) \omega t]
$$

with initial conditions $x_{2}(0)=0$ and $x_{2}^{\prime}(0)=0$. The solution of this equation is

$$
x_{2}(t)=\sum_{n=0}^{\infty} d_{2 n+1} \cos [(2 n+1) \omega t]
$$

Substituting Eq. (44) into Eq. (43) we obtain

$-\omega^{2} \sum_{n=0}^{\infty} 4 n(n+1) d_{2 n+1} \cos [(2 n+1) \omega t]=\frac{a_{1} a_{3} A^{3}}{8 \omega^{2}} \cos 3 \omega t-\frac{a_{3} A^{3}}{8 \omega^{2}} \sum_{n=1}^{\infty} c_{2 n+1} \cos [(2 n+1) \omega t]$

and the coefficients are

$$
d_{3}=\frac{a_{3}\left(c_{3}-a_{1}\right) A^{3}}{64 \omega^{4}}
$$




$$
\begin{gathered}
d_{2 n+1}=\frac{A^{3} a_{3} c_{2 n+1}}{32 n(n+1) \omega^{4}}, \quad n \geq 2 \\
d_{1}=-\sum_{n=1}^{\infty} d_{2 n+1}
\end{gathered}
$$

If we truncate the infinite series at Eq. (44), we obtain the following second-order approximate solution for $x_{2}$

$$
x_{2}^{(N)}(t)=d_{1}^{(N)} \cos \omega t+\sum_{n=1}^{N} d_{2 n+1} \cos [(2 n+1) \omega t]
$$

where

$$
d_{1}^{(N)}=-\sum_{n=1}^{N} d_{2 n+1}
$$

Eq. (49) has only a finite number of harmonics. It is possible to do this approximation because the absolute value of the coefficient $d_{2 n+1}$ decreases when $n$ increases as we can easily verify from Eqs. (39) and (47). Comparing Eqs. (44) and (49), and Eqs. (48) and (50), it follows that

$$
\lim _{N \rightarrow \infty} x_{2}^{(N)}(t)=x_{2}(t), \quad \lim _{N \rightarrow \infty} d_{1}^{(N)}=d_{1}
$$

As we are analyzing the second-order approximation we consider $N=2$ in Eqs. (49) and (50), in other words, only three harmonics $(n=0,1,2)$. Taking this into account, the approximate solution can be written as follows

$$
x_{2}^{(2)}(t)=d_{3}(\cos 3 \omega t-\cos \omega t)+d_{5}(\cos 5 \omega t-\cos \omega t)
$$


From Eqs. (46) and (47) the following expression for the coefficients $d_{3}$ and $d_{5}$ are obtained

$$
d_{3}=\frac{8 A^{3}}{21 \pi \omega^{4}}-\frac{A^{3}}{45 \pi^{2} \omega^{4}}, \quad d_{5}=\frac{8 A^{3}}{2025 \pi^{2} \omega^{4}}
$$

Therefore, the second-order approximation to the periodic solution is given by

$$
\begin{aligned}
x_{a 2}(t) & =x_{0}\left(t, \omega_{2}\right)+x_{1}^{(1)}\left(t, \omega_{2}\right)+x_{2}^{(2)}\left(t, \omega_{2}\right) \\
& =\left(A-b_{3}-d_{3}-d_{5}\right) \cos \left(\omega_{2} t\right)+\left(b_{3}+d_{3}\right) \cos \left(3 \omega_{2} t\right)+d_{5} \cos \left(5 \omega_{2} t\right) \\
& =0.97358 A \cos \left(\omega_{2} t\right)+0.025847 A \cos \left(3 \omega_{2} t\right)+0.000572875 A \cos \left(5 \omega_{2} t\right)
\end{aligned}
$$

where $\omega_{2}(A)$ is given by Eq. (42).

Following the same procedure used for calculating $\omega_{1}(A)$ and $\omega_{2}(A)$, we obtain the following expression for the third-order approximate frequency

$$
\omega_{3}(A)=0.914711 \sqrt{A}
$$

\section{Results and discussion}

We illustrate the accuracy of the modified approach by comparing the approximate solutions previously obtained with the exact frequency $\omega_{e x}$ and other results in the literature. In particular, we will consider the solution of Eq. (1) using the harmonic balance method $[6,41,44]$. This method is a procedure for determining analytical approximations to the periodic solutions of differential equations using a truncated Fourier series representation. Like the homotopy perturbation method, the harmonic balance method can be applied to nonlinear oscillatory problems where a linear term does not exist, the nonlinear terms are not small, and there is no perturbation parameter. However, it is very 
difficult to use the harmonic balance method to construct higher-order analytical approximations because this method requires solving analytical solutions of sets of algebraic equations with very complex nonlinearities.

The exact frequency is given by the following expression (see Appendix)

$$
\omega_{e x}(A)=0.914681 \sqrt{A}
$$

By applying harmonic balance method and using the first-order approximate solution

$$
x_{M 1}(t)=A \cos \omega t
$$

to Eq. (1), Mickens determined angular frequency, $\omega_{M 1}$, as [6]

$$
\omega_{M 1}(A)=\sqrt{\frac{8 A}{2 \pi}}=0.921318 \sqrt{A} \quad \text { Relative error }=0.73 \%
$$

Mickens [6, 44] also used the second-order harmonic balance approximation (generalized harmonic balance method)

$$
x_{M 2}(t)=\frac{A_{1} \cos \omega t}{1+B_{1} \cos 2 \omega t}
$$

to the periodic solution of Eq. (1) and the following equation is obtained for $B_{1}$

$$
\left(\frac{563}{28}\right) B_{1}^{3}+\left(\frac{149}{28}\right) B_{1}^{2}-\left(\frac{129}{7}\right) B_{1}-1=0
$$

Eq. (60) is the correct one when the generalized harmonic balance method is applied to Eq. (1) and not the following equation obtained by Mickens $[6,44]$

$$
\left(\frac{563}{28}\right) B_{1}^{3}+\left(\frac{338}{28}\right) B_{1}^{2}-\left(\frac{129}{7}\right) B_{1}-1=0
$$


From Eq. (60) we obtain

$$
B_{1}=-0.053602
$$

The angular frequency is given by the expression

$$
\omega_{M 2}(A)=\sqrt{\frac{32\left(17 B_{1}+7\right)\left(1+B_{1}\right) A}{105 \pi\left(9 B_{1}^{2}-12 B_{1}\right)}}
$$

and using the result of Eq. (62),

$$
\omega_{M 2}(A)=0.914044 \sqrt{A} \quad \text { Relative error }=0.070 \%
$$

From Eqs. (1), (59) and (62) we can obtain $A_{1}$, and its value is

$$
A_{1}=0.946398 A
$$

Substitution of all these results into Eq. (59) gives the following expression for the second-order harmonic balance approximate periodic solution of Eq. (1)

$$
x_{M 2}(t)=\frac{0.946398 A \cos \omega_{M 2} t}{1-0.053602 \cos 2 \omega_{M 2} t}
$$

The frequency values and their relative errors obtained in this paper applying a modified He's homotopy perturbation method are the following

$$
\begin{array}{cl}
\omega_{1}(A)=0.921318 \sqrt{A} & \text { Relative error }=0.73 \% \\
\omega_{2}(A)=0.914274 \sqrt{A} \quad \text { Relative error }=0.045 \%
\end{array}
$$




$$
\omega_{3}(A)=0.914711 \sqrt{A} \quad \text { Relative error }=0.032 \%
$$

It can be observed that these equations provide excellent approximations to the exact period regardless of the oscillation amplitude $A$. It is also clear that at the approximation order, the accuracy of the result obtained in this paper is better than those obtained previously by Mickens $[6,41,44]$.

The normalized exact periodic solution $x_{e x} / A$ achieved by numerically integrating Eqs. (1) and (2), and the proposed first and second order normalized approximate periodic solutions, $x_{a} / A$ in Eqs. (34) and (54), are plotted in Figures 1 and 2, respectively, while in Figures 3 and 4 we plotted the first and second order normalized solution obtained by harmonic balance method (Eqs. (57) and 66)). In these figures parameter $h$ is defined as follows

$$
h=\frac{2 \pi t}{\omega_{e x}(A)}
$$

Note that these figures are valid for all values of $A$. It can be observed that the second order approximate solution (Eq. (54)) gives excellent analytical approximate periodic solutions.

\section{Conclusions}

He's homotopy perturbation method has been used to obtain three approximate frequencies for an anti-symmetric, quadratic nonlinear oscillator for which the elastic force term is proportional to $x|x|$. Excellent agreement between approximate frequencies and the exact one has been demonstrated and discussed, and the discrepancy of the second-order approximate frequency, $\omega_{2}(A)$, with respect to the exact one is as low as $0.045 \%$. Furthermore, we concluded that discontinuous function had no tangible effect on the effectivity of the method. The general conclusion is that this modified homotopy perturbation method provides an easy and direct procedure for determining 
approximations to the periodic solutions of Eq. (1). This procedure also gives a very accurate estimate for the angular frequency at the second level of approximation. Finally, we think that the method has great potential and can be applied to other strongly nonlinear oscillators with non-polynomial terms.

\section{Appendix}

Calculation of the exact angular frequency, $\omega_{e x}(A)$, proceeds as follows. Integrating Eq. (1) and using the initial conditions in Eq. (2), we arrive at

$$
\frac{1}{2}\left(\frac{\mathrm{d} x}{\mathrm{~d} t}\right)^{2}+\frac{1}{3}|x| x^{2}=\frac{1}{3} A^{3}
$$

From the representation above, we can derive the exact period as follows

$$
T_{e x}(A)=4 \sqrt{\frac{3}{2}} \int_{0}^{A} \frac{\mathrm{d} x}{\sqrt{A^{3}-x^{3}}}
$$

The substitution $x=A u^{1 / 3}$ gives, after some simplifications

$$
T_{e x}(A)=\sqrt{\frac{8 A}{3}} \int_{0}^{1} u^{-2 / 3}(1-u)^{-1 / 2} \mathrm{~d} u
$$

which can be written as follows

$$
T_{e x}(A)=\sqrt{\frac{8 A}{3}} B\left(\frac{1}{3}, \frac{1}{2}\right)
$$

where $B(m, n)$ is the Euler beta function defined as follows [45] 


$$
B(m, n)=\int_{0}^{1} u^{m-1}(1-u)^{n-1} \mathrm{~d} u
$$

Equation (A4) can be finally written as

$$
T_{e x}(A)=\sqrt{\frac{8 A}{3}} \frac{\Gamma(1 / 3) \Gamma(1 / 2)}{\Gamma(5 / 6)}=\sqrt{\frac{8 \pi A}{3}} \frac{\Gamma(1 / 3)}{\Gamma(5 / 6)}
$$

where $\Gamma(z)$ is the Euler gamma function [46], $\Gamma(1 / 2)=\sqrt{\pi}$ and the following relation has been taken into account

$$
B(m, n)=\frac{\Gamma(m) \Gamma(n)}{\Gamma(m+n)}
$$

The exact frequency can be obtained as follows

$$
\omega_{e x}(A)=\frac{2 \pi}{T_{e}(A)}=\sqrt{\frac{8 \pi A}{3}} \frac{\Gamma(1 / 3)}{\Gamma(5 / 6)}=\sqrt{\frac{3 \pi}{2}} \frac{\Gamma(5 / 6)}{\Gamma(1 / 3)} \sqrt{A}=0.914681 \sqrt{A}
$$

\section{Acknowledgements}

This work was supported by the "Ministerio de Educación y Ciencia", Spain, under project FIS2005-05881-C02-02, and by the "Generalitat Valenciana”, Spain, under project ACOMP/2007/020. 


\section{Figure captions}

Figure 1. Comparison of the approximate first-order periodic normalized solution, Eq. (34) (dashed line), by homotopy perturbation method with the exact solution (continuous line).

Figure 2. Comparison of the approximate second-order periodic normalized solution, Eq. (54) (dashed line), by homotopy perturbation method with the exact solution (continuous line).

Figure 3. Comparison of the approximate first order periodic normalized solution, Eq. (57) (dashed line), by harmonic balance method with the exact solution (continuous line).

Figure 4. Comparison of the approximate second order periodic normalized solution, Eq. (66) (dashed line), by generalized harmonic balance method with the exact solution (continuous line). 


\section{References}

[1] J. H. He, "A new perturbation technique which is also valid for large parameters", $J$. Sound Vib. 229, 1257-1263 (2000).

[2] J. H. He, "Modified Lindstedt-Poincare methods for some non-linear oscillations. Part III: double series expansion”, Int. J. Non-linear Sci. Numer. Simulation 2, $317-$ 320 (2001).

[3] T. Özis and A. Yildirim, "Determination of periodic solution for a $u^{1 / 3}$ force by He's modified Lindstedt-Poincaré method", J. Sound Vib. 301, 415-419 (2007).

[4] P. Amore and F. M. Fernández, "Exact and approximate expressions for the period of anharmonic oscillators", Eur. J. Phys. 26, 589-601 (2005).

[5] P. Amore, A. Raya and F. M. Fernández, "Alternative perturbation approaches in classical mechanics", Eur. J. Phys. 26, 1057-1063 (2005).

[6] R. E. Mickens, Oscillations in Planar Dynamics Systems (World Scientific, Singapore 1996).

[7] A. Beléndez, A. Hernández, A. Márquez, T. Beléndez and C. Neipp, "Analytical approximations for the period of a simple pendulum", Eur. J. Phys. 27, 539-551 (2006).

[8] A. Beléndez, A. Hernández, T. Beléndez, M.L. Álvarez, S. Gallego, M. Ortuño and C. Neipp "Application of the harmonic balance method to a nonlinear oscillator typified by a mass attached to a stretched wire", J. Sound Vib. 302, 1018-1029 (2007).

[9] G. R. Itovich and J. L. Moiola, "On period doubling bifurcations of cycles and the harmonic balance method", Chaos, Solitons \& Fractals 27, 647-665 (2005). 
[10] D. H. Shou and J. H. He, "Application of parameter-expanding method to strongly nonlinear oscillators", Int. J. Non-linear Sci. Numer. Simulation 8 (1), 121-124 (2007).

[11] J. H. He and X. H. Wu, "Construction of solitary solution and compact on-like solution by variational iteration method", Chaos, Solitons \& Fractals 29, 108-113 (2006).

[12] J. H. He, "Variational approach for nonlinear oscillators", Chaos, Solitons \& Fractals 34, 1430-1439 (2007).

[13] M. Tatari and M. Dehghan, "The use of He's variational iteration method for solving a Fokker-Planck equation”, Phys. Scr. 74, 310-316 (2006).

[14] M. Tatari and M. Dehghan, "Solution of problems in calculus of variations via He's variational iteration method", Phys. Lett. A 362, 401-406 (2007).

[15] B. Batiha, M. S. M. Noorani and I. Hashim, "Numerical simulation of the generalizaed Huxley equation by He's variational iteration method", Appl. Math. Comput. 186, 1322-1325 (2007).

[16] A. Beléndez, A. Hernández, T. Beléndez, A. Márquez and C. Neipp, “An improved 'heuristic' approximation for the period of a nonlinear pendulum: linear analysis of a classical nonlinear problem", Int. J. Non-linear Sci. Numer. Simulation 8 (3), 329334 (2007).

[17] Lei Geng and Xu-Chu Cai, "He's frequency formulation for nonlinear oscillators", Eur. J. Phys. 28, 923-931 (2007).

[18] L. Xu, "He's parameter-expanding methods for strongly nonlinear oscillators", $J$. Comput. Appl. Math. 207, 148-154 (2007). 
[19] J. H. He, Non-perturbative methods for strongly nonlinear problems Dissertation (De-Verlag im Internet GmbH, Berlin 2006).

[20] J. H. He, "Some asymptotic methods for strongly nonlinear equations", Int. J. Mod. Phys. B, 20, 1141-1199 (2006).

[21] J. H. He, "The homotopy perturbation method for nonlinear oscillators with discontinuities", Appl. Math. Comp. 151, 287-292 (2004).

[22] X. C. Cai, W. Y. Wu and M. S. Li, "Approximate period solution for a kind of nonlinear oscillator by He's perturbation method", Int. J. Non-linear Sci. Numer. Simulation 7 (1), 109-117 (2006).

[23] L. Cveticanin, "Homotopy-perturbation for pure nonlinear differential equation", Chaos, Solitons \& Fractals 30, 1221-1230 (2006).

[24] S. Abbasbandy, “Application of He's homotopy perturbation method for Laplace transform", Chaos, Solitons \& Fractals 30, 1206-1212 (2006).

[25] A. Beléndez, A. Hernández, T. Beléndez and A. Márquez, "Application of the homotopy perturbation method to the nonlinear pendulum”, Eur. J. Phys. 28, 93-104 (2007).

[26] A. Beléndez, A. Hernández, T. Beléndez, E. Fernández, M. L. Álvarez and C. Neipp, "Application of He's homotopy perturbation method to the Duffing-harmonic oscillator", Int. J. Non-linear Sci. Numer. Simulation 8 (1), 79-88 (2007).

[27] D. D. Ganji and A. Sadighi, "Application of He's homotopy-perturbation method to nonlinear coupled systems of reaction-diffusion equations", Int. J. Non-linear Sci. Numer. Simulation 7 (4), 411-418 (2006). 
[28] S. Abbasbandy, “A numerical solution of Blasius equation by Adomian's decomposition method and comparison with homotopy perturbation method", Chaos, Solitons \& Fractals 31, 257-260 (2007).

[29] A. Siddiqui, R. Mahmood and Q. Ghori, "Thin film flow of a third grade fluid on moving a belt by He's homotopy perturbation method", Int. J. Non-linear Sci. Numer. Simulation 7 (1), 15-26 (2006).

[30] J. H. He, "Homotopy perturbation method for solving boundary value problems", Phys. Lett. A 350, 87-88 (2006).

[31] M. Rafei and D. D. Ganji, "Explicit solutions of Helmhotlz equation and fifth-order $\mathrm{KdV}$ equation using homotopy perturbation method", Int. J. Non-linear Sci. Numer. Simulation 7 (3), 321-328 (2006).

[32] D. D. Ganji, “The application of He's homotopy perturbation method to nonlinear equations arising in heat transfer", Phys. Lett. 355, 337-341 (2006).

[33] M. S. H. Chowdhury and I. Hashim, “Application of homotopy-perturbation method to nonlinear population dynamics models", Phys. Lett. A 368, 251-258 (2007).

[34] P. D. Ariel and T. Hayat, "Homotopy perturbation method and axisymmetric flow over a stretching sheet", Int. J. Non-linear Sci. Numer. Simulation 7 (4), 399-406 (2006).

[35] M. S. H. Chowdhury and I. Hashim, "Solutions of time-dependent Emden-Fowler type equations by homotopy-perturbation method", Phys. Lett. A 368, 305-313 (2007).

[36] M. S. H. Chowdhury and I. Hashim, "Solutions of a class of singular second-order IVPs by homotopy-perturbation method", Phys. Lett. A 365, 439-447 (2007). 
[37] F. Shakeri and M. Dehghan, "Inverse problem of diffusion by He's homotopy perturbation method", Phys. Scr. 75, 551-556 (2007).

[38] M. Dehghan and F. Shakeri, "Solution of a partial differential equation subject to temperature overspecification by He's homotopy perturbation method", Phys. Scr. 75, 778-787 (2007).

[39] M. S. H. Chowdhury and I. Hashim "Solutions of Emden-Fowler equations by homotopy-perturbation method", Nonlinear Analysis B: Real World Applications (accepted for publication).

[40] L. Cveticanin, "Application of homotopy-perturbation to non-linear partial differential equations", Chaos, Solitons \& Fractals (2007), doi:10.1016/j.chaos.2007.07.053.

[41] R. E. Mickens and I. Ramadhani, "Investigations of an anti-symmetric quadratic non-linear oscillator", J. Sound Vib. 155, 190-193 (1992).

[42] S-Q. Wang and J. H. He, "Nonlinear oscillator with discontinuity by parameterexpansion method", Chaos, Solitons \& Fractals (2007), doi:10.1016/ j.chaos.2007.07.055.

[43] A. Beléndez, C. Pascual, S. Gallego, M. Ortuño and C. Neipp, “Application of a modified He's homotopy perturbation method to obtain higher-order approximations of a $x^{1 / 3}$ force nonlinear oscillator", Phys. Lett. A (2007), doi:10.1016/ j.physleta.2007.06.042.

[44] R. E. Mickens and M. Mixon, “Application of generalized harmonic balance method to an anti-symmetric quadratic non-linear oscillator", J. Sound Vib. 159, 546-568 (1992). 
[45] M. Abramowitz and I. A. Stegun (Eds.), "Beta Function" and "Incomplete Beta Function" $\$ 6.2$ and 6.6 in Handbook of Mathematical functions with Formulas, Graphs and Mathematical Tables. New York: Dover, pp. 258 and 263, 1972.

[46] M. Abramowitz and I. A. Stegun, (Eds.), "Gamma (Factorial) Function" and "Incomplete Gamma Function" 6.1 and 6.5 in Handbook of Mathematical functions with Formulas, Graphs and Mathematical Tables. New York: Dover, pp. 258 and 263, 1972. 
FIGURE 1

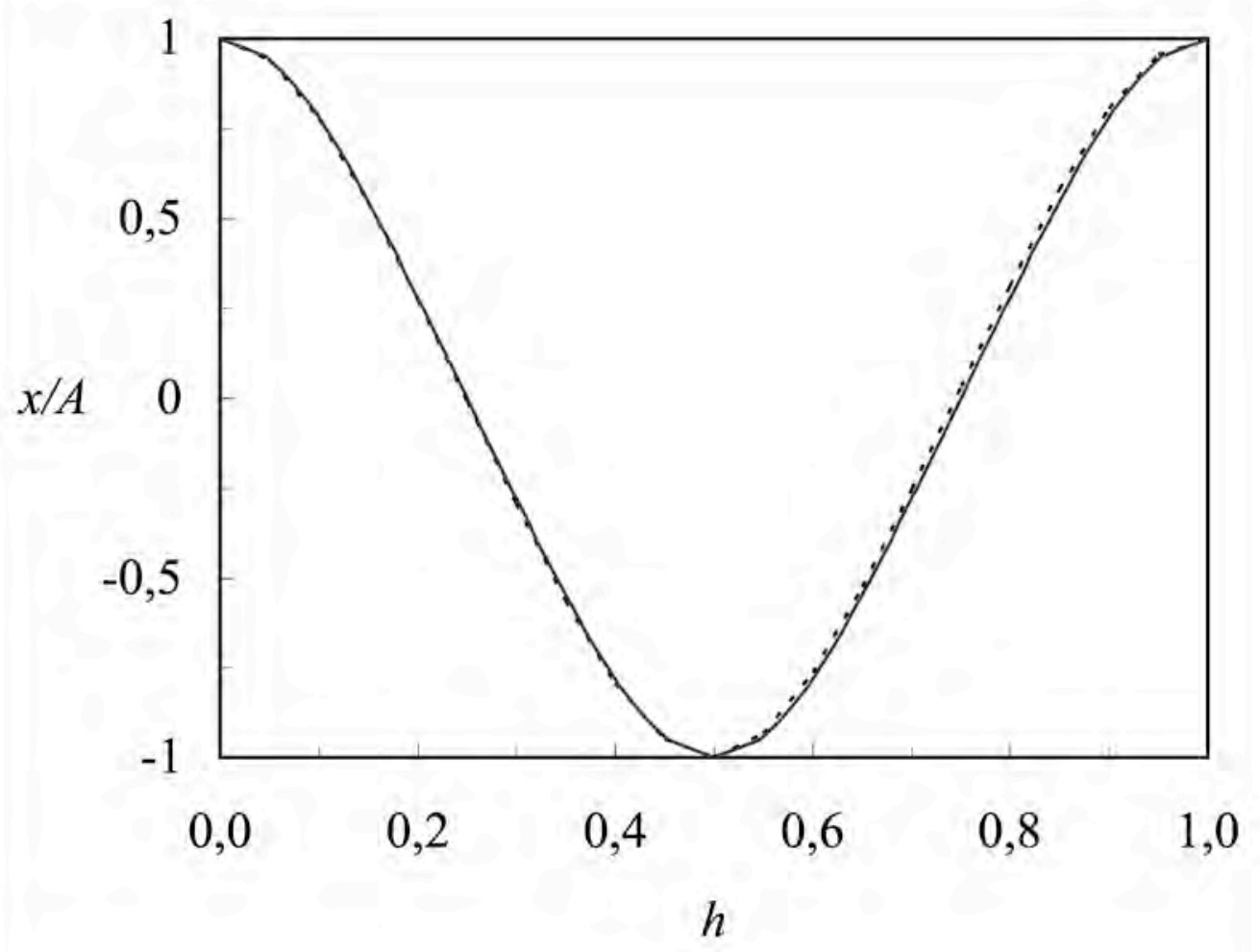


FIGURE 2




FIGURE 3

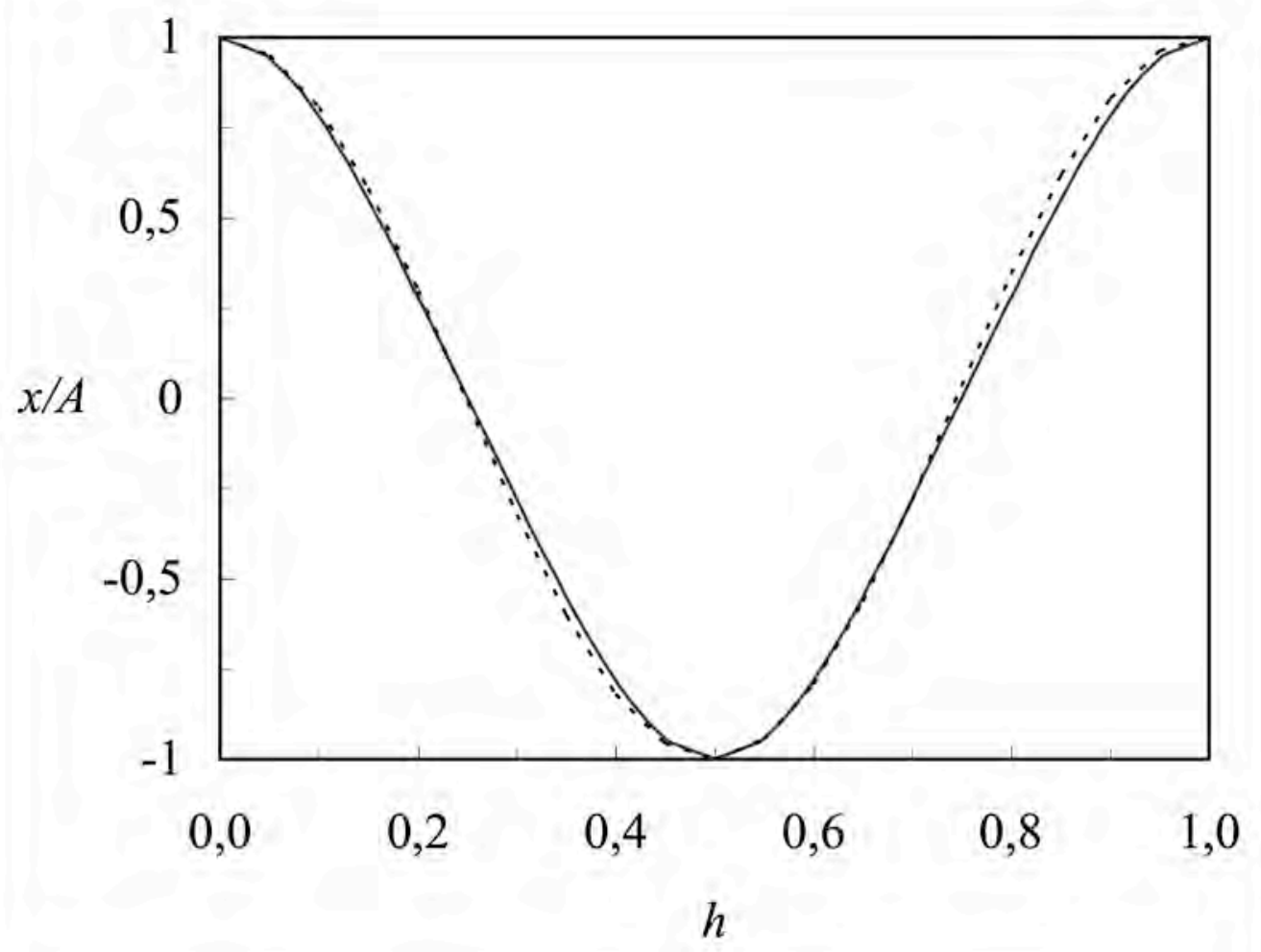


FIGURE 4




